\title{
Correction of Atmospheric Refraction Errors in Radio Height Finding*
}

\author{
W. B. Sweezy and B. R. Bean
}

\section{Contribution from Central Radio Propagation Laboratory, National Bureau of Standards, Boulder, Colo.}

(Received August 11, 1962; revised October 11, 1962)

\begin{abstract}
Atmospheric refraction errors in height finding radars are studied by means of detailed refraction calculations for a wide range of meteorological conditions. For targets up to 70,000 feet above ground and 150 miles ground distance from the radar site, the mean height error was found to be as much as 5,000 feet with a standard deviation of 1,200 feet. A correction for the surface value of the refractive index at the radar site would eliminate the mean height error and reduce the maximum standard deviation to less than 900 feet. An additional correction for the initial gradient of the refractive index and the value of the refractive index at one kilometer above the surface would reduce the maximum standard deviation to less than 400 feet. Methods of correcting height errors based on available meteorological data are presented and shown to be operationally practical.
\end{abstract}

\section{Introduction}

As a radio ray passes through the atmosphere the length and direction of its path varies with the radio refractive index. Uncorrected radar output determines the position of a target by assuming a straight-line path at constant velocity. The difference between the straight path and the actual path results in an error which becomes increasingly significant as the distance to the target increases. The height error (the component of the position error normal to the surface of the earth) constitutes over 95 percent of the total error. Until recently, the range of height inding equipment was sufficiently limited so that the refraction errors could be either neglected, or approximated by a constant effective earth's radius correction [Schelleng, Burrows, and Ferrell, 1933].

Bauer, Mason, and Wilson [1958] obtained an equation for accurately estimating radar target heights in a specific exponential atmosphere. Beckmann [1958] presented a probability estimate of the height errors without using meteorological measurements.

The purpose of the study is to investigate the correlation between available meteorological parameters and height errors for targets of interest in terminal air traffic control and to develop height error correction procedures using these parameters. The height errors for various target positions relative to the radar site are correlated with meteorological parameters measured at or above the site to determine the predictability of height errors independently of target position. The correction procedures are developed to account for atmospheric variations and target position by combining the meteorological and geometric considerations.

\section{Background}

\subsection{Refractive Index}

The radio refractive index, $n$, of a propagation medium is the ratio of the free-space velocity of light, $c$, to the velocity in the medium, $v$, (i.e., $n=c / v$ ). Since the propagation velocity of the atmosphere is only slightly less than the free-space velocity, it is often convenient to use the scaled-up difference between the refractive index and unity. This quantity is called the refractivity and is denoted by $N=(n-1) \times 10^{6}$.

The refractivity is obtained from meteorological parameters by

$$
N=77.6 \frac{P}{T}+3.73 \times 10^{5} \frac{e}{T^{2}},
$$

where $P$ is the total atmospheric pressure in millibars, $T$ is the absolute temperature, and $e$ is the water vapor pressure in millibars. Normally, the equation for $N$ is dominated by the first term so that the refractivity can be approximated by an exponential function of height as shown by Bean and Thayer [1959a].

\subsection{Ray Theory}

If the gradient of refractive index is assumed to be normal to the surface of the smooth spherical earth and

$$
\frac{d n}{d r}>-\frac{1}{r}
$$

then, for frequencies greater than $100 \mathrm{kc} / \mathrm{s}$, the path of a radio ray is determined by Snell's law for polar coordinates:

\footnotetext{
*This work was sponsored in part by the Federal Aviation Agency.
} 


$$
n r \cos \theta=n_{0} r_{0} \cos \theta_{0},
$$

where $\theta$ is the local elevation angle of the ray, and $r$ is distance from the center of the earth to a point on the ray as shown in figure 1 . The bending angle, $\tau$, is determined by [Smart, 1931]

$$
\tau=-\int_{r_{0}}^{r_{0}+h} \frac{\cot \theta}{n} \frac{d n}{d r} d r .
$$

The distance, $d$, along the surface of the earth is obtained by

$$
d=r_{0} \phi=r_{0}\left(\tau+\theta-\theta_{0}\right) .
$$

The length of the path is called the geometric range and is obtained by

$$
R=\int_{r_{0}}^{r_{0}+h} \csc \theta d r
$$

and the apparent or radio range is found by

$$
R_{e}=\int_{r_{0}}^{r_{0}+h} n \csc \Theta d r=R+\int_{r_{0}}^{r_{0}+h} N \times 10^{-6} \csc \theta d r .
$$

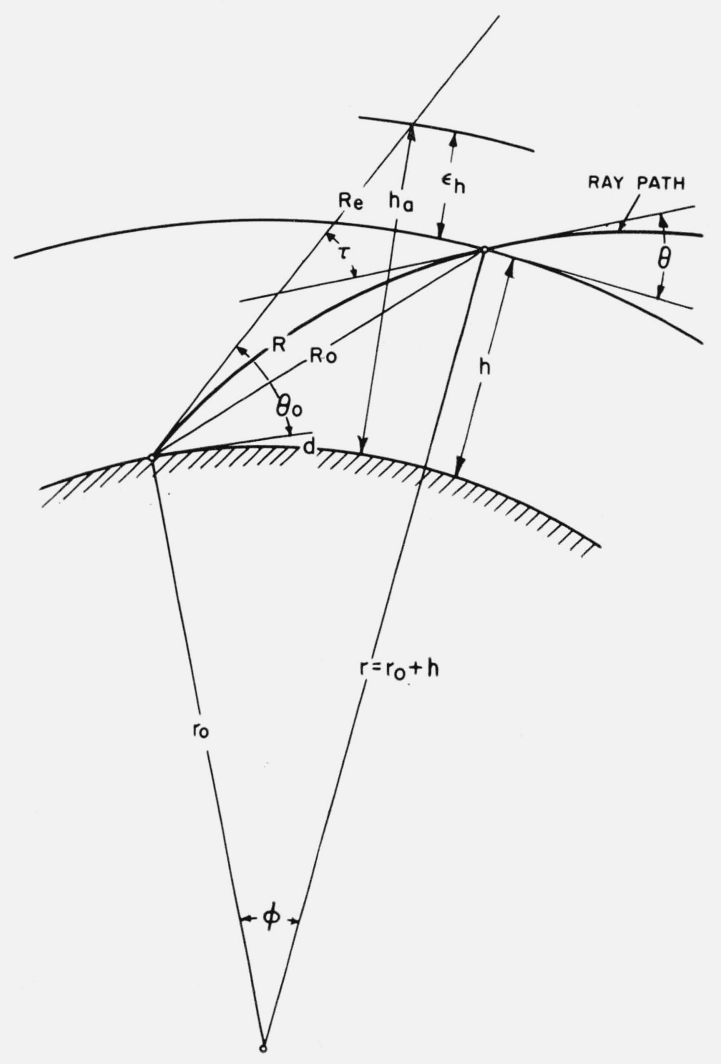

Figure 1. Geometry of radio ray refraction.
Because the difference between $R_{e}$ and the true slant range, $R_{0}$ is extremely small compared to the height error; the slant range and radio range are assumed to be identical to the geometric range, $R$.

The apparent height of the target, in figure 2 , is obtained by solving

$$
\left(r_{0}+h_{\mathrm{a}}\right)^{2}=r_{0}^{2}+R^{2}+2 r_{0} R \sin \theta_{0}
$$

for $h_{a}$. The following form is useful for numerical calculations:

$$
h_{a}=\frac{R\left(R+2 r_{0} \sin \Theta_{0}\right)}{r_{0}+\sqrt{r_{0}^{2}+R\left(R+2 r_{0} \sin \Theta_{0}\right)}} .
$$

The height error for a target at height, $h$, is found by

$$
\epsilon_{h}=h_{a}-h,
$$

which will always be positive if $n$ decreases with height.

If the refractive index is known as a function of height, the foregoing procedure is useful for determining the height error when the true height and the arrival angle of the ray are hypothesized. Unfortunately, it is not applicable for obtaining the height error from the apparent position of the target.

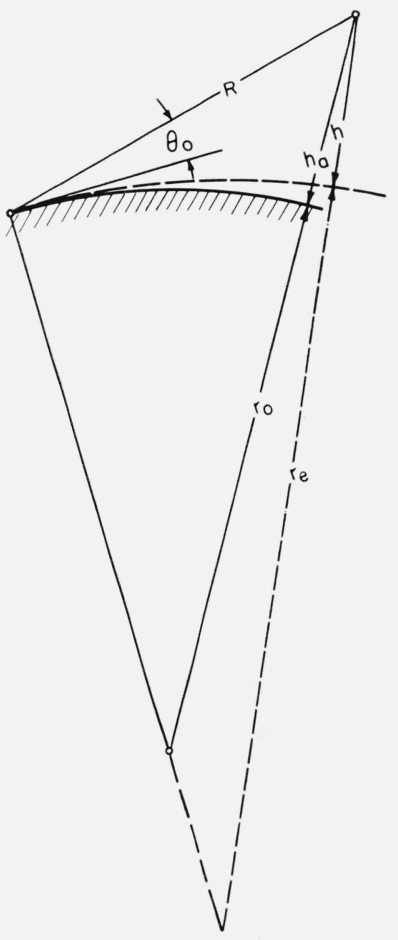

Figure 2. Effective earth's radius geometry. 


\subsection{Effective Earth's Radius}

The inaccuracy of a constant effective earth's radius correction stems mainly from the assumption that all radio rays have the same constant curvature. The accuracy would be greatly enhanced if an "average" effective radius could be determined for each ray path.

The following expression, with the effective earth's radius denoted by $r_{e}$ in figure 2 .

$$
\left(r_{e}+h\right)^{2}=r_{e}^{2}+R^{2}+2 r_{e} R \sin \Theta_{0},
$$

can be combined with (6) and (8) to obtain

$$
\epsilon_{h}\left(1+\frac{h_{a}+h}{2 r_{e}}\right)=\left(\frac{R^{2}-h_{a}^{2}}{2}\right)\left(\frac{1}{r_{0}}-\frac{1}{r_{e}}\right) .
$$

Because the expression multiplying $\epsilon_{h}$ in (10) differs from unity by less than $4 \times 10^{-3}$ for all target heights ( $h \leq 70,000 \mathrm{ft})$ and ranges $(R \leq 155$ miles) to be considered, the height error can be approximated as

$$
\epsilon_{h} \simeq\left(\frac{R^{2}-h_{a}^{2}}{2}\right)\left(\frac{1}{r_{0}}-\frac{1}{r_{e}}\right),
$$

with an error never in excess of 0.4 percent. The difference between the curvature of the actual earth and the curvature of the "average" effective earth for the ray path (i.e., $1 / r_{0}-1 / r_{e}$ ) represents the "average" curvature of the ray. Thus, if the ray curvature can be determined as a function of the target position and the refractive index structure, (11) provides a simple formula for approximating the height error.

The curvature of a ray, $K$, at any point on the path is expressed by [Millington, 1957]

$$
K=-\frac{1}{n} \frac{d n}{d r} \cos \theta
$$

or, from (1), replacing $r$ by $r_{0}+h$,

$$
K=-\frac{n_{0} \cos \theta_{0}}{n^{2}\left(1+h / r_{0}\right)} \frac{d n}{d h}
$$

From (6), ignoring the term of the order $1 / r_{0}^{2}$, one obtains

$$
\cos ^{2} \theta_{0} \simeq\left(1+\frac{h_{a}}{r_{0}}\right)\left(\frac{R^{2}-h_{a}^{2}}{R^{2}}\right),
$$

so that (13) becomes

$$
K \simeq-\frac{n_{0}}{n^{2}} \frac{\left(R^{2}-h_{a}^{2}\right)^{1 / 2}}{R} \frac{\left(1+\frac{h_{a}}{r_{0}}\right)^{1 / 2}}{\left(1+\frac{h}{r_{0}}\right)} \frac{d n}{d h}
$$

The refractive index usually decreases with height so that the quantity

$$
\frac{n_{0}}{n^{2}} \frac{\left(1+\frac{h_{a}}{r_{0}}\right)^{1 / 2}}{1+\frac{h}{r_{0}}} \simeq 1
$$

varies only slightly with height, and the curvature at a point on the ray path can be approximated by

$$
K \simeq \frac{\left(R^{2}-h_{a}^{2}\right)^{1 / 2}}{R}\left|\frac{d n}{d h}\right|
$$

Therefore, (11) becomes

$$
\epsilon_{h} \simeq \frac{\left(R^{2}-h_{a}^{2}\right)^{3 / 2}}{2 R} g,
$$

where $g$ represents an average gradient on the ray path as defined in the following section.

Since $g$ depends upon the meteorological conditions along the path, the basic problem is to determine $g$ for a given target from the conditions at and/or near the surface.

\section{Procedure}

\subsection{Meteorological Parameters}

Measurement of the refractivity at the radar site will provide an estimate of the gradient if a model of the refractive index structure is assumed. In the exponential model, for example,

$$
n(h)=1+N_{s} \exp (-c h) \times 10^{-6},
$$

where $N_{s}$ is the surface refractivity and $c$ is a constant, the gradient

$$
\frac{d n}{d h}=-c N_{s} \exp (-c h) \times 10^{-6}
$$

For a target at a height $h_{t}$ the simple average gradient along the ray path from radar to target is

$$
g=-\frac{1}{h_{t}} \int_{0}^{h_{t}} \frac{d n}{d h} d h
$$

which for the exponential model is

$$
g=\frac{N_{s}}{h_{t}}\left[1-\exp \left(-c h_{t}\right)\right] \times 10^{-6},
$$

but, since $h_{t}$ is not known, $g$ must be approximated as a function of the apparent height.

Additional meteorological measurements at a sufficient height above the surface to obtain values significantly different from the surface values can be used to determine the initial gradient of refractivity,

$$
G_{0}=\left.\frac{d N}{d h}\right|_{h=0}
$$


assuming the initial layer to be exponential yields

$$
G_{0}=\frac{N_{s}}{H} \log \left(\frac{N_{H}}{N_{s}}\right),
$$

where $N_{H}$ is the refractivity at the height, $H$, in kilometers of the above surface measurements. The initial gradient provides a boundary condition for estimating $g$ as a function of the apparent height. The average gradient for the ray path determined with the initial gradient and the true height for the exponential model is

$$
g=\frac{G_{0}}{c h_{t}}\left[\exp \left(-c h_{t}\right)-1\right] \times 10^{-6} .
$$

For the purposes of this study the average (per kilometer) gradient of the first kilometer of the atmosphere is the only prediction parameter used which will require upper air measurements. The average 1-km gradient,

$$
\Delta N=N_{1}-N_{s},
$$

where $N_{1}$ is refractivity at $1 \mathrm{~km}$ above the surface, was selected because climatological summaries [Bean, Horn, and Ozanich, 1960] can be used to estimate the height error when meteorological measurements are unobtainable.

\subsection{Calculation and Correlation of Height Errors}

Bean, Cahoon, and Thayer [1960] selected refractive index profiles, determined from radiosonde observations at thirteen climatically distinct locations, which represent a wide variety of mutually exclusive profile types. This profile sample was used for the present study because it represents a complete range of meteorological conditions. The ray paths at arrival angles varying from 0 to near $90^{\circ}$ were determined for each profile by numerical evaluation of (1) through (5) using methods similar to those described by Bean and Thayer [1959b]. The height errors were calculated with (7) and (8) at selected height intervals to $70,000 \mathrm{ft}$ for each ray path. Newton's method of interpolation with divided differences was used to determine height errors for fixed ground distances to 150 miles. The limits of height and distance were chosen to extend bevond the current needs in terminal air traffic control, but are sufficiently restricted to allow some of the previous assumptions.

The prediction parameters, $N_{s}, G_{0}$, and $\Delta N$, were obtained from each of the refractive index profiles. Linear and multiple regression analyses were employed to obtain least squares estimates of the height error at each height and distance for each prediction parameter and for various combinations of the parameters.

\subsection{Estimation of the Average Gradient}

Based on the correlations, the following forms suggested by (18) were selected for approximating $g$ :

$$
\begin{gathered}
g_{1}=\frac{N_{s}}{h_{a}} f_{11}\left(h_{a}\right), \\
g_{2}=\frac{N_{s}}{h_{a}} f_{21}\left(h_{a}\right)+\frac{G_{0}}{h_{a}} f_{22}\left(h_{a}\right),
\end{gathered}
$$

or

$$
g_{3}=\frac{N_{s}}{h_{a}} f_{31}\left(h_{a}\right)+\frac{G_{0}}{h_{a}} f_{32}\left(h_{a}\right)+\frac{\Delta N}{h_{a}} f_{33}\left(h_{a}\right),
$$

where $g_{1}$ is an estimate of the average gradient if only surface observations are available, $g_{2}$ is an improved estimate obtained with additional tower measurements, and $g_{3}$ is an estimate obtained with the addition of upper air measurements such as radiosonde observations.

To obtain a direct estimate of the height error, (21) through (23) were combined with (17), and the functions $f_{i j}(i \geq j=1,2,3)$ were determined as least squares polynomials.

\section{Results}

\subsection{Regression Analysis}

The volume of data processed is of sufficient magnitude that it is impractical to include it all in this report. Therefore, certain information obtained from the regression analysis was selected as being the most significant.

The mean height error is representative of average meteorological conditions, and, therefore, provides the best general estimate obtainable if meteorological data are not available at the radar site under consideration. In figure 3, the mean height error was plotted for each target position and then contour lines were drawn to display the mean height error as a function of the true height and distance.

The standard deviation (about the mean) of the height errors provides a measure of the residual error if the mean is used as an estimate, since 68 percent of the observed height errors are expected to be within \pm 1 standard deviation of the mean height error if the observations are normally distributed. The standard deviation is displayed as a function of target position in figure 4 . The construction of figure 4 and subsequent figures is similar to that of figure 3.

The standard error of estimate establishes the same confidence limits for prediction with a regression as the standard deviation does for the mean. Thus, the standard error provides a measure of the residual error if the height errors are estimated by a regression equation involving meteorological parameters. The standard error of estimate was determined for each of the following regression equations

and

$$
\begin{gathered}
\epsilon_{h}=b_{1} N_{s}+a, \\
\epsilon_{h}=b_{1} N_{s}+b_{2} G_{0}+a,
\end{gathered}
$$

$$
\epsilon_{h}=b_{1} N_{s}+b_{2} G_{0}+b_{3} \Delta N+a .
$$




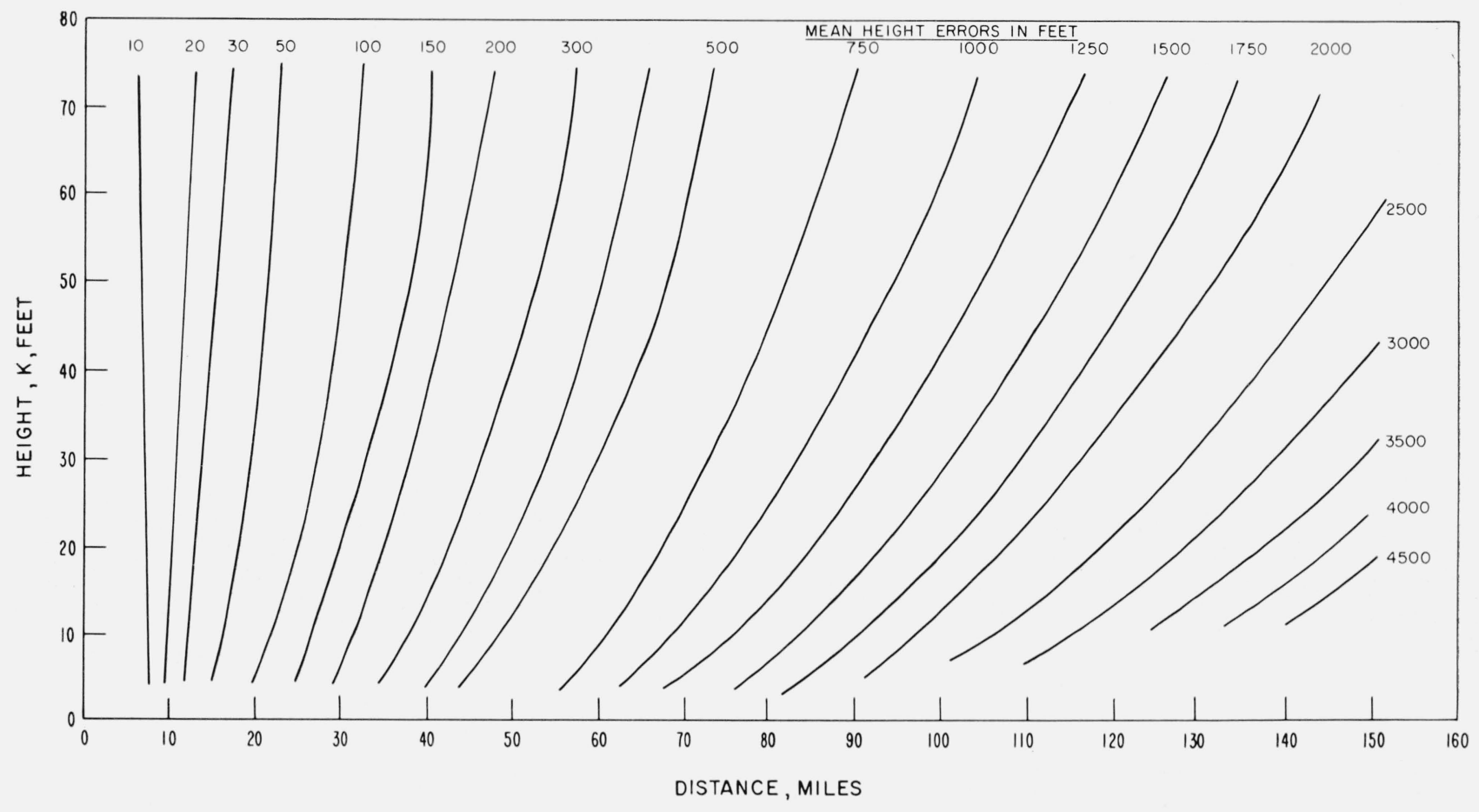

Figure 3. Mean height errors in feet.

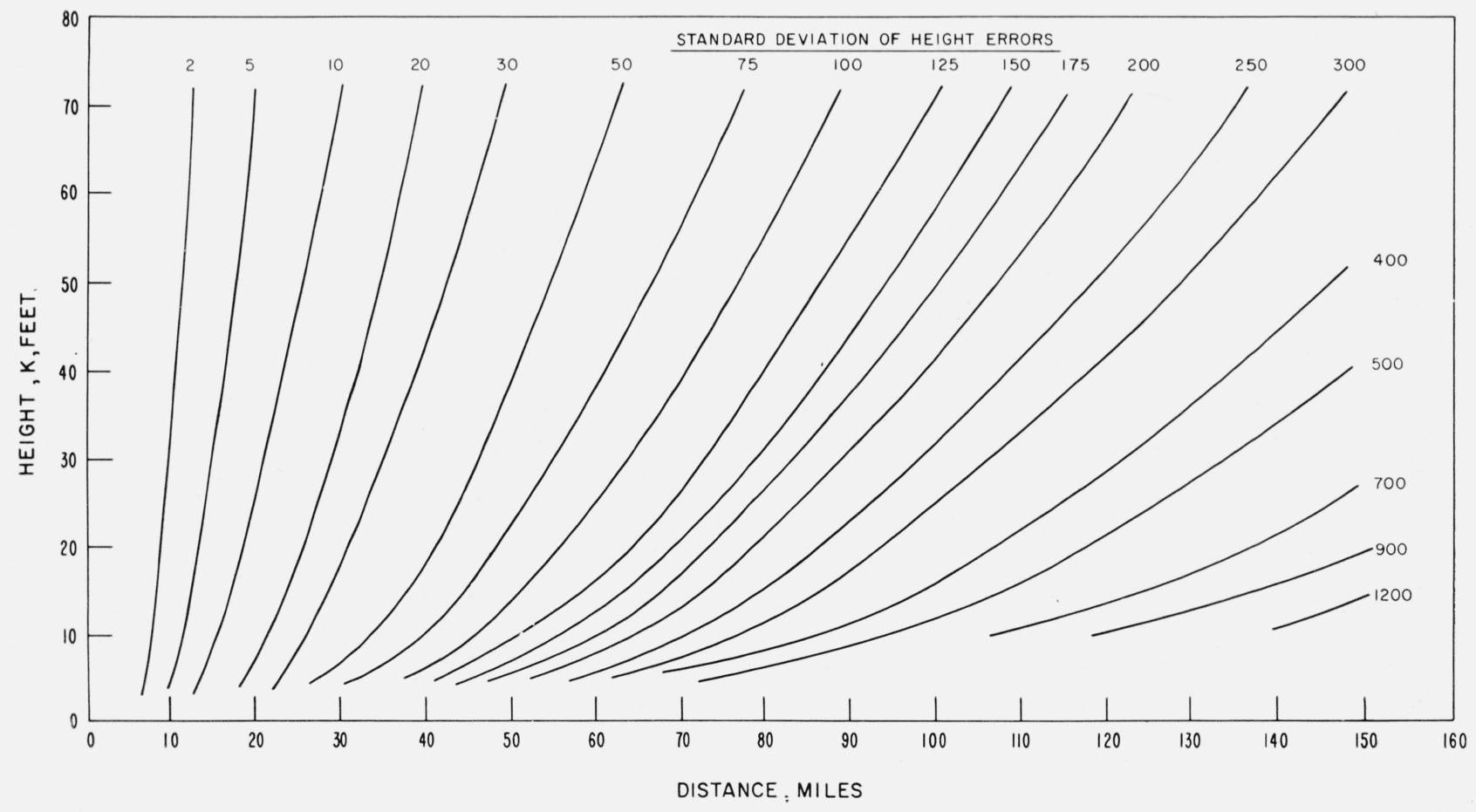

Figure 4. Standard deviation of height errors. 
The standard error of estimate with (24) is displayed in figure 5. Comparison of figures 4 and 5 indicates the improvement, that is, the reduction in residual error, if surface meteorological observations are used in place of the mean to predict the height error.
The standard errors of estimate with (25) and (26) are shown in figures 6 and 7 , respectively. These figures demonstrate how each additional parameter, obtained from tower or upper air measurements, enhances the accuracy of the estimate.

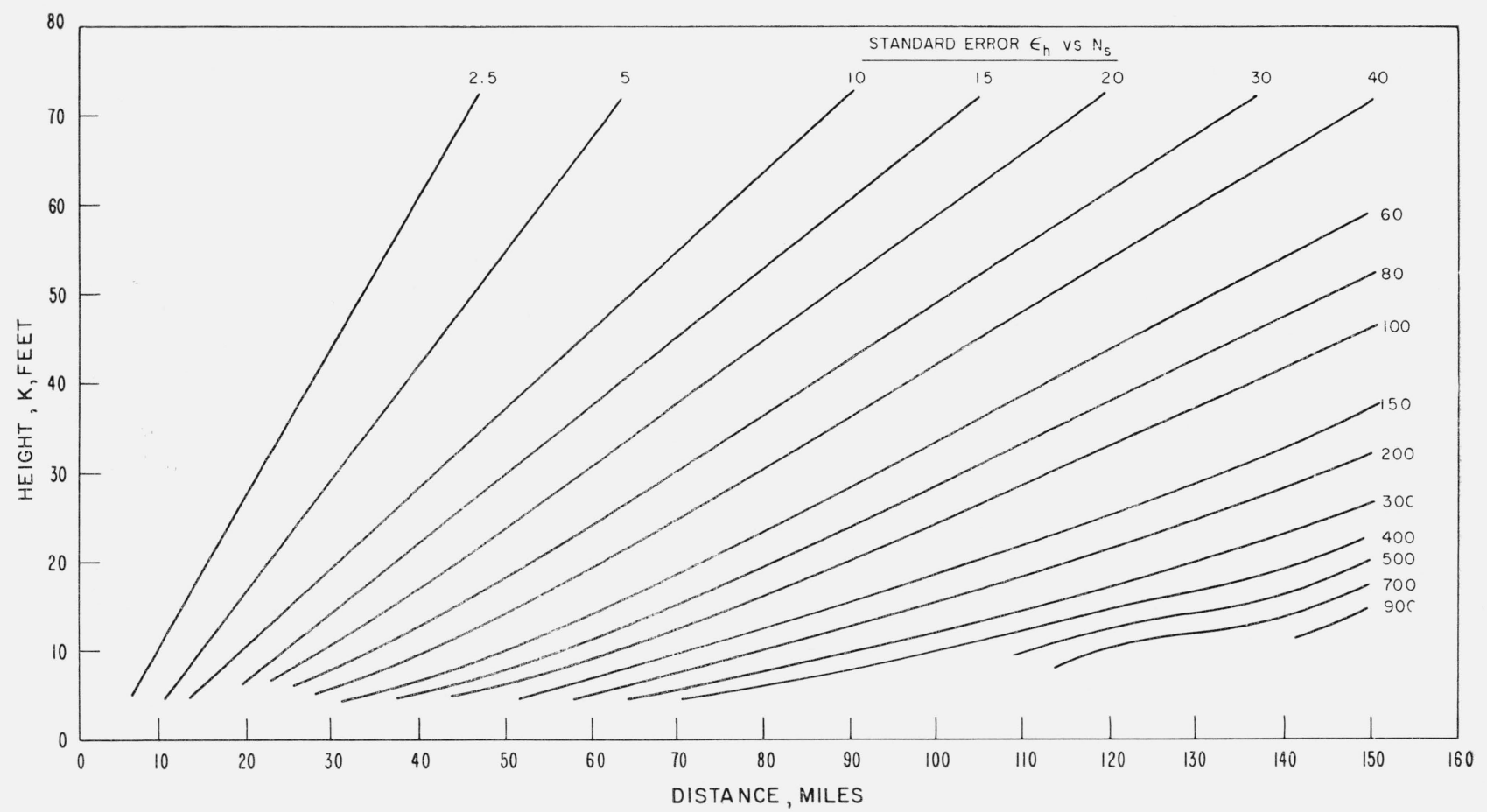

Figure 5. Standard error $\epsilon_{\mathrm{h}}$ versus $N_{\mathrm{B}}$.

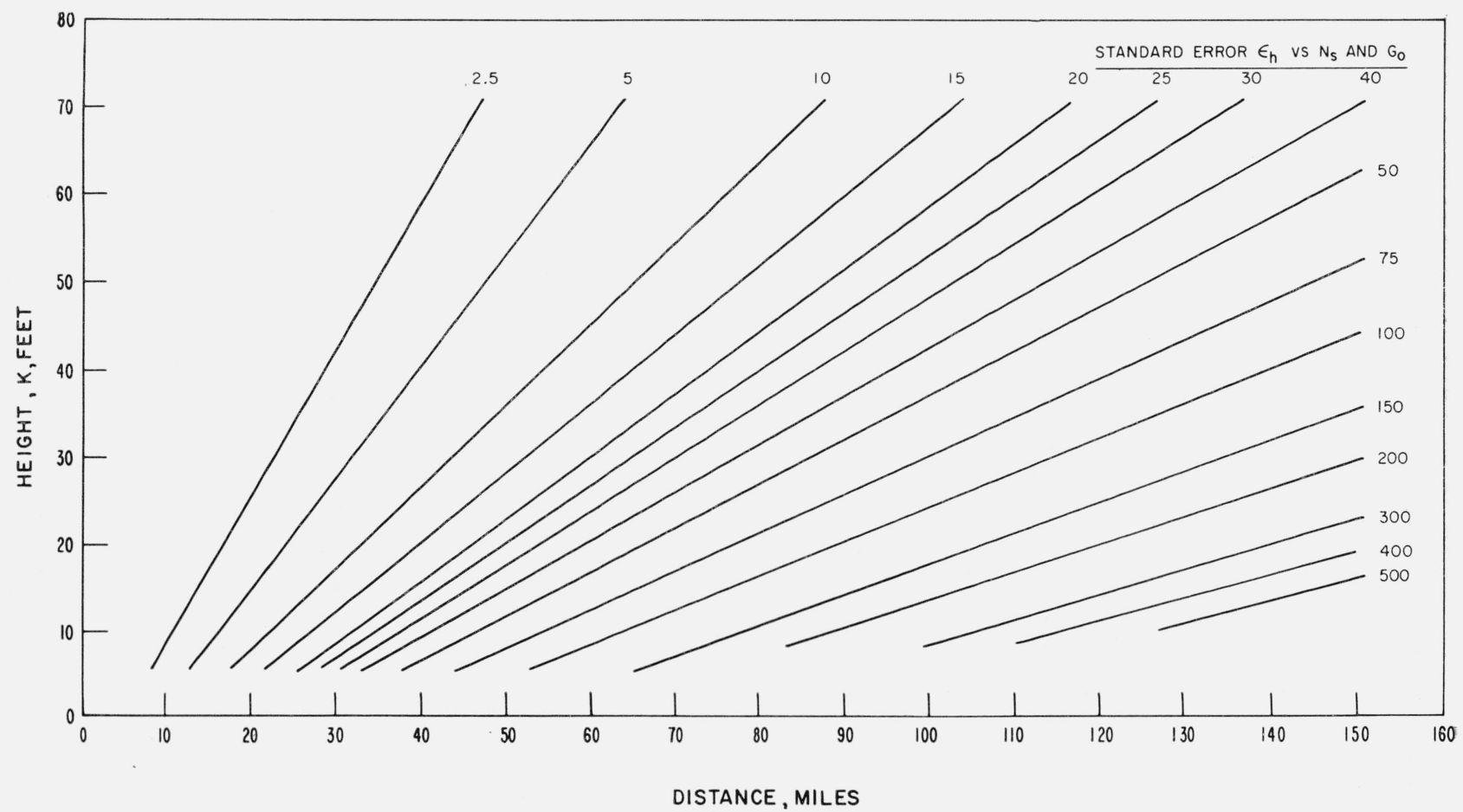

Figure 6. Standard error $\epsilon_{\mathrm{h}}$ versus $N_{\mathrm{s}}$ and $G_{0}$. 


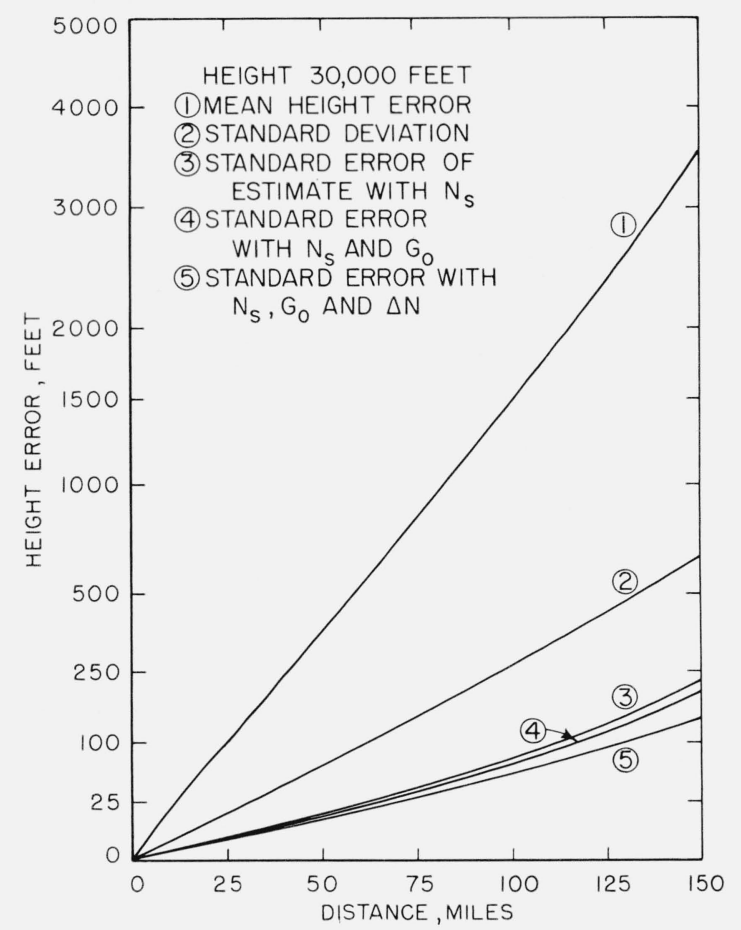

FIgure 9. Height error statistics for fixed height of 30,000 feet.

\subsection{Height-Error Equations}

The equations for approximating $\boldsymbol{\epsilon}_{h}$ were determined as

$$
\begin{aligned}
\epsilon_{h_{1}} & =a_{10} \frac{D}{h_{a}{ }^{2}}+\frac{D^{3 / 2}}{R} g_{1}+k, \\
\epsilon_{h_{2}} & =a_{20} \frac{D}{h_{a}{ }^{2}}+\frac{D^{3 / 2}}{R} g_{2}+k, \\
\epsilon_{h_{3}} & =a_{30} \frac{D}{h_{a}{ }^{2}}+\frac{D^{3 / 2}}{R} g_{3}+k,
\end{aligned}
$$

where

$$
D=R^{2}-0.03587 h_{a}{ }^{2},
$$

$g_{1}, g_{2}$, and $g_{3}$ are obtained from (21) through (23) with

$$
\begin{aligned}
& f_{i 1}\left(h_{a}\right)=a_{i 1}+a_{i 2} h_{a}+a_{i 3} h_{a}{ }^{2} \quad(i=1,2,3), \\
& f_{i 2}\left(h_{a}\right)=a_{i 4}+a_{i 5} h_{a} \quad(i=2,3),
\end{aligned}
$$

$$
f_{33}\left(h_{a}\right)=a_{36}+a_{37} h_{a},
$$

for $R$ in miles and $h_{a}$ in thousands of feet. The term in $D / h_{a}^{2}$ was introduced to account for, in part, a large negative constant term which tended to produce negative height errors for ranges less than 30 miles. Furthermore, the inclusion of this term increased the accuracy of the estimate of $\epsilon_{h i}$ by about 2 percent. An additional term in $h_{a}^{3}$ for (30) increased the accuracy by about 1 percent but introduced a fictitious minimum near $60,000 \mathrm{ft}$, while a term in $h_{a}^{2}$ for (31) and (32) increased the accuracy of (28) and (29) by less than 0.1 percent. The relative improvement of (28) over (27) is about 3 percent and of (29) over (28) about 1 percent.

The coefficients $a_{i j}$ are listed in table $\mathrm{A}$. The constant term, $k$, which would vanish if the equations were exact, is about -70 for a least squares approximation.

\section{Conclusions}

Height-error correction can be significantly improved by accounting for the surface refractivity at the radar site. The use of the initial gradient, in addition to the surface refractivity, yields a significant improvement only for targets beyond about 60 miles and below $15,000 \mathrm{ft}$. In this case, $G_{0}$ is important not only to improve the accuracy but to determine if the assumption in section 2.2 has been violated, namely, if $G_{0} \leq-10^{6} / r_{0}$. The still further improvement obtained with the use of $\triangle N$ would not, in general, justify the trouble and expense of measuring this parameter.

If the distance to the target exceeds about 50 miles, the normal decrease with height of the gradient should be accounted for in a height error correction.

\section{Appendix. Coefficients for the Regres- sion Equations}

Constant term, $a$ :

Equation (24) Table 1

Equation (25) Table 2

Equation (26) Table 3

Coefficient of $N_{s}, b_{1}$ :

Equation (24) Table 4

Equation (25) Table 5

Equation (26) Table 6

\begin{tabular}{|c|c|c|c|c|c|c|c|c|}
\hline & 0 & 1 & 2 & 3 & 4 & 5 & 6 & 7 \\
\hline $\begin{array}{l}1 \\
2 \\
3\end{array}$ & $\begin{array}{l}-19.596 \\
-17.849 \\
-15.319\end{array}$ & $\begin{array}{r}0.014096 \\
.011202 \\
.006388\end{array}$ & $\begin{array}{r}0.77906 \times 10^{-4} \\
.13665 \times 10^{-3} \\
.18549 \times 10^{-3}\end{array}$ & $\begin{array}{r}0.67545 \times 10^{-6} \\
.58925 \times 10^{-7} \\
.39074 \times 10^{-7}\end{array}$ & $\begin{array}{r}-0.64975 \times 10^{-2} \\
-.55818 \times 10^{-2}\end{array}$ & $\begin{array}{r}0.12340 \times 10^{-3} \\
.12671 \times 10^{-3}\end{array}$ & -0.023980 & $-0.22547 \times 10^{-4}$ \\
\hline
\end{tabular}

Coefficient of $G_{0}, b_{2}$ :

Equation (25) $\quad$ Table 7

Equation (26) Table 8

Coefficient of $\Delta N, b_{3}$ :

Equation (26) Table 9

TABLE A. Coefficients $\mathrm{a}_{\mathrm{ij}}$ 
TABLE 1

HEIGHT KFT

DISTANCE
MILES

MILES

5
10
15
20
25
30
35
40
45
50
60
70
80
90
100
110
120
130
140
150

TABLE 2

DI STANCE

MILES
5
10
15
20
25
30
35
40
45
50
60
70
80
90
100
110
120
130
140
150

10

$\begin{array}{ll}-3 . & -3 . \\ -14 . & -13 . \\ -31 . & -29 . \\ -55 & -53\end{array}$

$\begin{array}{ll}-31 . & -29 . \\ -55 . & -53 .\end{array}$

$-86$.

$-123$.

$-166$.

$-215$.

$-269$.

$-327$.

$-586$.

-709 .

$-83$.

-119.
-162.

$-211$.

$-267$.

$-329$.

$-470$.

$-633$.

$-812$.

$-1186$.

$-1352$.

$-1473$.
15

$-2$

-10.
-24.

$-44$.

$-69$.

-100 .

-136.
-178.

-226 .

-280 .

$-404$.

$-552$.

$-722$.

$-913$.

$-1122$.

-1342.
-1565.

-1565.
-1774.

$-1946$.

-2048 .
20

25

-1 .

-8.
-19.

$-19$.

$-56$.

-81 .

$-111$.

-146.
-186.

-231 .

$-335$.

-461 .

-607 .

$-776$.

$-965$.

$-1176$.

$-1404$.

-1644 .

$-1889$.

$-2123$.

\begin{aligned} & 25 \\ &-- \\ &-6 \\ &-15 \\ &-28 \\ &-45 \\ &-66 \\ &-91 \\ &-120 \\ &-153 \\ &-190 \\ &-277 \\ &-381 \\ &-505 \\ &-648 \\ &-812 \\ &-997 \\ &-1203. \\ &-1429 \\ &-1672 \\ &-1928 \\ & \hline\end{aligned}

30

35

40

50

60

70

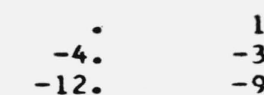

$-12$.

$-23$.

-37.
-54.

-54.
-74.

$-98$.

$-125$.

$-156$.

-228.
-315.

-418 .

$-539$.

$-678$.

-836.
-1014.

-1014.
-1215.

$-1434$.

-1674 .

2

-9.
-18.

$-29$.

-44.
-60.

-80 .

$-102$.

-127.
-187.

$-259$.

$-345$.

-446.
-563

$-697$.

-850 .

$-1022$.

$-1214$.

1
-2
-7
-14
-24
-35
-49
-65
-83
-104
-153
-212
-284
-367
-465
-578
-707
-852
-1019
-1203

-4.
-9.

-9.
-15.

$-23$.

$-32$.

-43.
-55.

$-70$.

$-103$.

$-144$.

$-193$.

$-252$.

-320 .

$-399$.

-491 .

$-597$.

-718 .

$-855$.

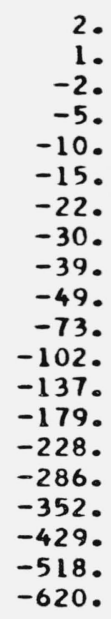

2.

$-1$.

-1.
-3.
-7.

$-11$.

-16 .

$-22$.

$-29$.

$-36$.

$-54$.

$-103$.

$-134$.

$-171$.

$-171$.

$-264$.

$-322$.

$-389$.

$-465$.

HEIGHT KFT

5

10

15

20

25

30

35

40

\begin{tabular}{rr}
-3. & -3. \\
-12. & -12. \\
-28. & -29. \\
-50. & -51. \\
-77. & -80. \\
-110. & -115. \\
-148. & -156. \\
-190. & -204. \\
$-235 \circ$ & -257. \\
-284. & -316. \\
-383. & -449. \\
-473. & -600. \\
-537. & -762. \\
. & -925. \\
. & -1076. \\
. & -1189. \\
. & -1236. \\
. &. \\
\hline &.
\end{tabular}

$-2$.

-10.
-24.

$-43$.

$-67$.

$-97$.

$-133$.

$-174$.

$-221$.

$-273$.

-394.
-536.

-536.
-700.

$-880$.

$-1075$.

$-1275$.

$-1468$.

-1637 .

$-1748$.

-1
-8

$-19$.

-35 .

$-55$.

$-80$.

$-110$.

$-144$.

$-183$.

$-227$.

$-329$.

-452.
-595.

$-757$.

$-940$.

$-1141$.

$-1355$.

$-1576$.

$-1794$.

$-1989$.

-6.
-6.
-15.
-28.
-45.
-65.
-90.
-118.
-151.
-187.
-273.
-376.
-497.
-637.
-797.
-976.
-1174.
-1390
-1619.
-1855

-4
-12
-22
-36
-53
-73
-97
-124
-154
-225
-311
-413
-531
-667
-822
-996
-1189
-1400
-1630

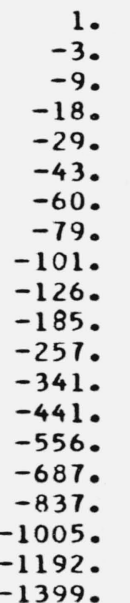

1
-2
-7
-14
-23
-35
-48
-64
-82
-103
-151
-210
-281
-363
-460
-571
-697
-840
-1003
-1183

50

60

70

$\begin{array}{rrr}2 . & 2 . & 2 . \\ -4 & 1 . & 1 \\ -4 . & -2 . & -1 . \\ -9 . & -5 . & -3 . \\ -15 . & -10 . & -7 . \\ -23 . & -15 . & -11 . \\ -32 . & -22 . & -16 . \\ -43 . & -30 . & -22 . \\ -55 . & -38 . & -28 . \\ -69 . & -48 . & -36 . \\ -102 . & -72 . & -54 . \\ -143 . & -101 . & -76 . \\ -191 . & -136 . & -102 . \\ -249 . & -178 . & -133 . \\ -317 . & -226 . & -170 \\ -395 . & -283 . & -212 . \\ -486 . & -349 . & -262 . \\ -590 . & -425 . & -319 . \\ -708 . & -512 . & -385 . \\ -843 . & -612 . & -460\end{array}$


HEIGHT KFT

OISTANCE
MILES
5
10
15
20
25
30
35
40
45
50
60
70
80
90
100
110
120
130
140
150

TABLE 4

HEIGHT KFT

DISTANCE
MILES
5
10
15
20
25
30
35
40
45
50
60
70
80
90
100
110
120
130
140
150

5

15

20

25

30

35

40

50

60

70

.0318
.1153
.2545
.4495
.7005
1.0078
1.3714
1.7917
2.2688
2.8030
4.0426
5.5119
7.2127

.0313
.1059

.0296

.0281

.0267

.0257

.0705

.1454

.0248

.0652

.1326

.2271

. 2025

.1795

.1608

.4046

.6291

.9040

.5494

.3123

.4835
.6934

.2784

.4300

.6159

1.0728

.9424

.2504

.3858
.5519

.7489

.3489

.4983

1.0923

1.2311

1.0923

2.0336

1.4018

1.5599

1.9295
2.7938

2. 1968

1. 7113

2.4780

2.4780
3.3981

4.3513

3. 8300

5.0453

4.4788

7.2966

6.4464

5.7273

8.2133

9.0736

8.0412

7.1529

8.7649

12.2529

14.5240

11.0533

13.2284

15.5836

9.8363

11.8360

14.0399

10.5679
12.5772

- 9774

.6756

1. 5305

2. 2155

3. 0380

4. 0046

5.1225

6.4005

7.8482

9.4723

14.7931

13.3002

19.0333

17.2171

15.5220

1.1154

1. 3788

1. 9952

2. 7355

3. 6053

4.6119

5.7634

7.0684

8. 5370

10.1777

12.0051

.0243

.0608

0236

.0543

.023

.0498

.1218

.1056

.0943

.2074

.1776

.1568

.2373

.4531

2704

.3841

.5190

.7998

.6754

.3359

.4529

.5885

7429

.8536

1.0119

1.2503

1.0539

- 7429

.9163
1.3216

2. 4788

1.5223

1.3216
1.8072

2. 3769

3.2666

2.7442

3. 0342

5.2207

3. 5078

3.7846

4.3804

3.7846
4.6329

7.7337

5.3687

4.6329
5.586

7.7254

6.6512

10.8816

9.1125

7.8354

9.1488

$-14$.

$-14$.

-19.
-25.

-31 .

$-47$.

$-66$.

$-88$

$-115$.

$-146$.

$-182$.

$-224$

.

-327.
-389.

14.0251

10.6504

.0225 
TABLE 5

HEIGHT KF T

DI STANCE
MILES

5

10

15

20

25

30

35

40

50

60

70

$\begin{array}{cc} & \text { MILES } \\ & 5 \\ & \\ & 10 \\ & 15 \\ & 20 \\ & \\ & 25 \\ & 30 \\ & 35 \\ & 40 \\ & 45 \\ & 50 \\ & 60 \\ & 70 \\ & 80 \\ & 90 \\ & 100 \\ & 110 \\ & 120 \\ & 130 \\ & 140 \\ & 150 \\ & \end{array}$

.0285
.1014
.2226
.3915
.6072
.8686
1.1741
1.5217
1.9089
2.3325
3.2717
4.2984
5.3577
.
.
.

.0303

.0292

.0922
.1972

.0279

.0835

.0267

.0762

.1588

.0257

.0701

.0249

.1763
.3065

.2746

.03440

.3862

.5997

.8603
1.1680

1.5225

1.9235

.5340
.7661

1. 0411

1. 3591

1.7204

2.1254

2.3701

76

4.5945
5.9423

7. 4110

3.0671

4. 1841

5.4790

6. 9435

8.9558

10.5049
11.9716

.

8.5676

10.3305

12.1954
14.1047

15.9660

.4742

.6797

.9234
1.2056

1.5268

1.8873

2.7287

3. 7339

4.9073

0.2511
7.7696

9.4588

11.3115

13.3104

15.4244

.4239
.6069

.8240

1.0755

1.3619

1.6836

2. 4355

3. 3361

4.3907

5.6047

6.9854

8. 5356

10.2579

12.1560

14.2189

$-1441$

.0649

.0244

.0607

.0543

.0232

.0498

.1053

.0942

.1212

1768

.3817

.5457

.3459

.4939

.3155

- 4497

.2690

.1563

.2365

.3821

$\begin{array}{ll}.7403 & .6694 \\ .9658 & .8729\end{array}$

.793

.5162

.3346

.5860

1.0037

1.2227

1.1046

8486

$1.5114 \quad 1.365$

2.1864

2. 9957

1.9743
2.7052

1. 2400

1. 7928

2.4560

1. 5126

.7396

.9121

3.5629

5.0400

4.5537

3.2348

2.7249

1.798

6.2887

5.6849

7.6975

9.2713

11.0200

12.9405

6.9635

8.3974

5.1625

3.4816

2.3643

3.0171

4.3456

6.3258

7.6323

1.7614

10.7087

5.3231

3.7620

4.6035

5.5484

7.6489

6.6027

13.7056

12.4899

9.0139
10.5245

7.773

0227

.0463

.0857

.1410

.2122

.24028

.5226

.6589

.8119

1.1691

1.5965

2.0968

2.6730

3.3294

4. 0699

4.8989
5.8234

6.0477

7.9804

TABLE 6

HEIGHT KFT

DISTANCE

10

15

20

25

30

35

40

50

60

70

.0091

.0231

.0259

.0265

.0720

.0265

.0687

.0262

.0652

.0260

.0619

1218

.0255

.0540

058

.2214

.2058

.3138

.4462

.4821

.3902
.5567

.7532

. 3646

.5198
.7033

.9151

.9358

.0291

1.2939

1.5863
2.2485

2. 9993

3.8207

4.6780

5.5316

6. 3291

7.0067

7.4937

7.7293

1.2354

1.5206
2.1771

2.9439

3.8126

1.1553

1.4239

2. 0459

2.7804

.6030

.7843

1.0712

1. 3209

1. 9009

2.5892

3.6248

3.3863

4.7689

5.7994

6.8710

7.9475

4.5741

5.6251

6.7588

7.9627

8.9769

9.2092

4.2912

5.3042

6.4182

7.6279

8.9193

$10.4569 \quad 10.2723$

10.6160

11.6542

11.6633

.9903

1.2213
1.7588

2.3986

3.1423

3.9913

4.9474

6.0094

7. 1738

8.4423

.1141

.1016
.1683

.2912

.2542

.3595

.5579

.4843

.6287

.7253

.9155

1. 1290

1. 6263

.7929

.9772

1.4070

1.9202

2.9101

2.5191

3.2075

50

3.9877

4.8629

7.9028 
TABLE 7

HEIGHT KF I

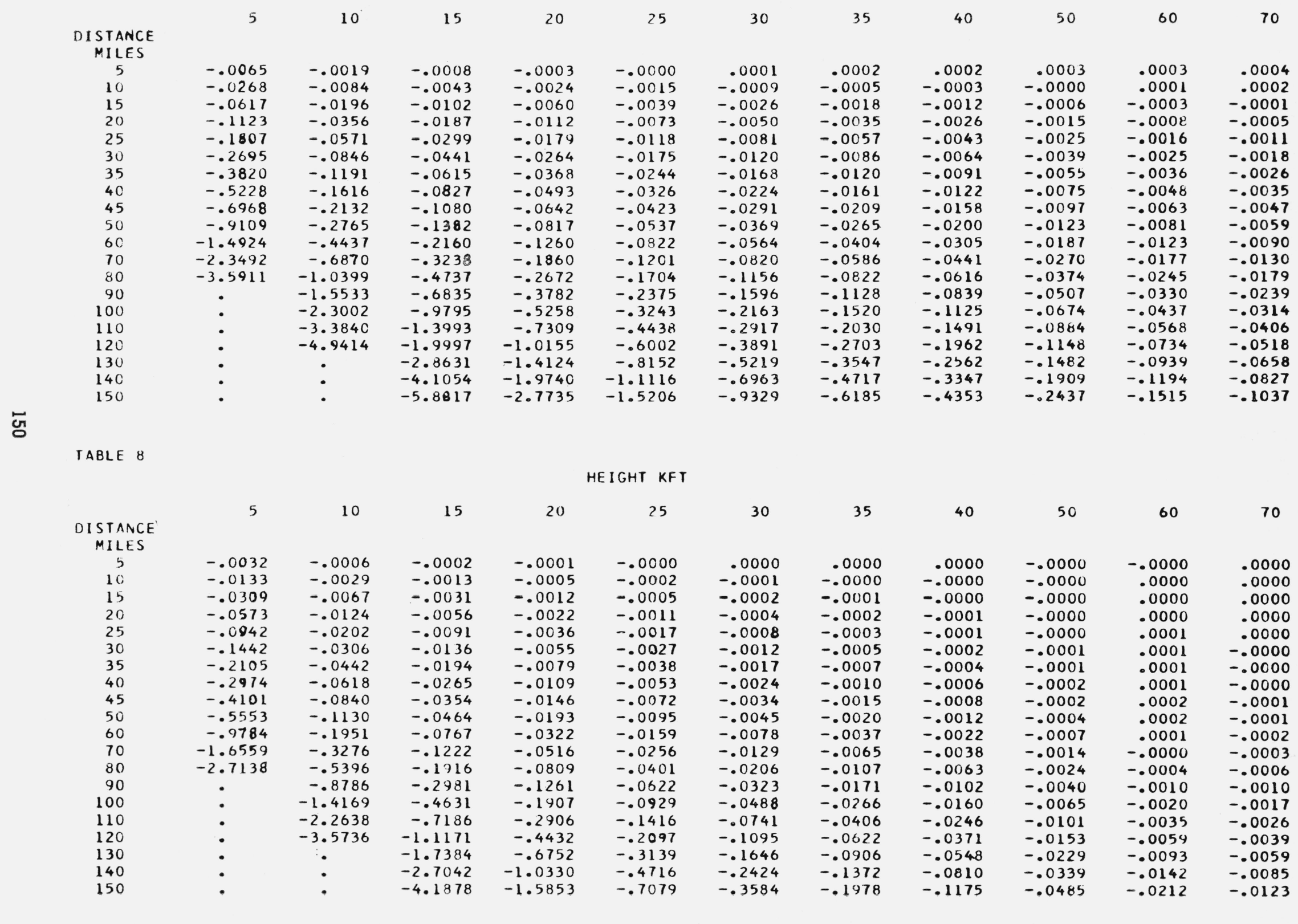




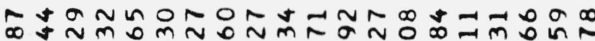

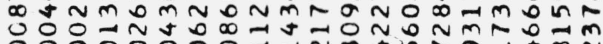

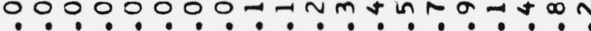
$i$ i i i i i i i i $-\dot{1}-\dot{1}$

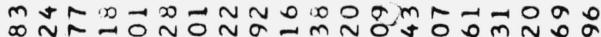

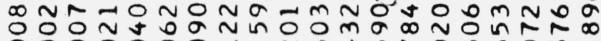
$0000000 \rightarrow \pi n m+n \pi 0 m 00$ nn - i i i i i i i i i- $\dot{i} \dot{\sim}$

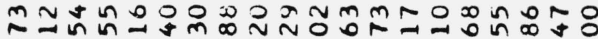

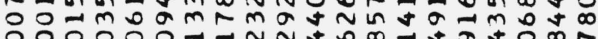

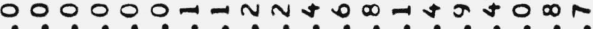
i $i$ i i i i i i

인

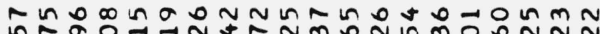

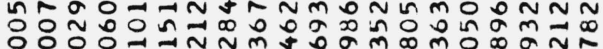
- $i$ i i i i i i

nn

ษิ ปN

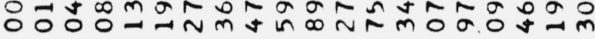

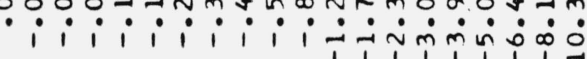
i 1 1111111

तี

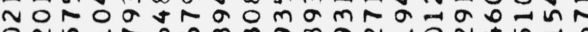

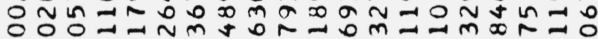
- $i$ i $i$ i $\dot{i} \dot{\sim} \dot{m} \dot{0} \dot{0} \dot{0}$

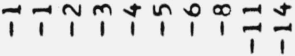

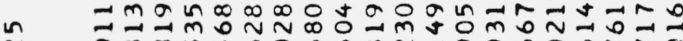

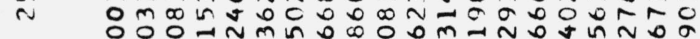

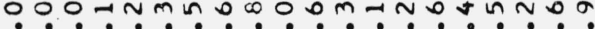

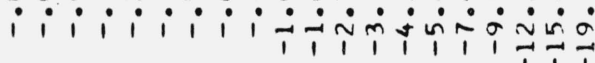

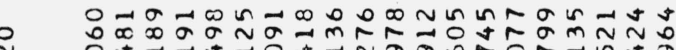

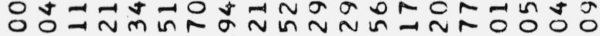

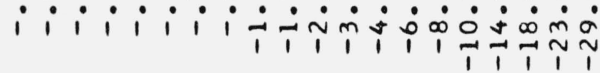

n

mat?

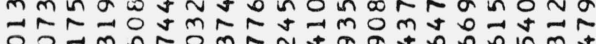

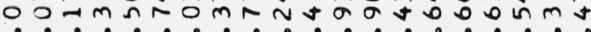

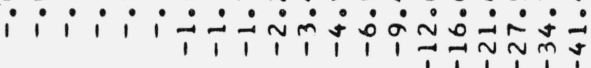

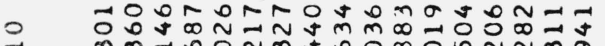

m

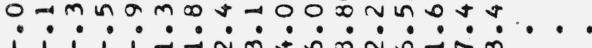

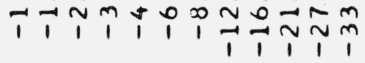

in

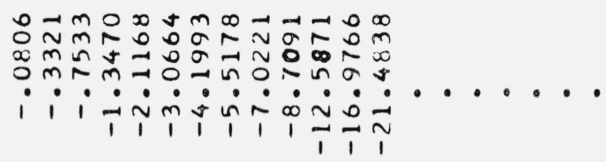

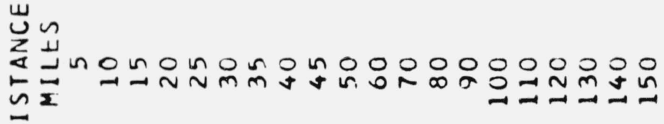

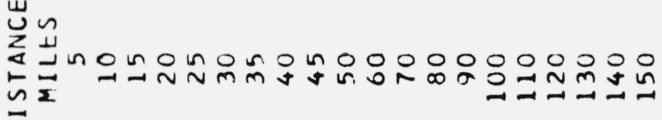

\section{References}

Bauer, J. R., W. C. Mason, and F. A. Wilson (August 27, 1958), Radio refraction in a cool exponential atmosphere, Tech. Report No. 186, Mass. Inst. Technol., Lincoln Laboratory

Bean, B. R., B. A. Cahoon, and G. D. Thayer (March 16, 1960), Tables for the statistical prediction of radio ray bending and elevation angle error using surface values of the refractive index, NBS Tech. Note 44.

Bean, B. R., and G. D. Thayer (1959a), Models of the atmospheric radio refractive index, Proc. IRE 47, No. 5, 740-755.

Bean, B. R., and G. D. Thayer (October 29, 1959b), CRPL exponential reference atmosphere, NBS Mono. No. 4.

Bean, B. R., J. D. Horn, and A. M. Ozanich, Jr. (November $25,1960)$, Climatic charts and data of the radio refractive index of the United States and the world, NBS Mono. No. 22 .

Beckmann, P. (1958), Height errors in radar measurements due to propagation causes, Acta Tech. 3, No. 6, 471-488.

Millington, G. (January 1957), The concept of the equivalent radius of the earth in tropospheric propagation, The Marconi Review Ro, No. 126, 79-93.

Schelleng, J. D., C. R. Burrows, and E. B. Ferrell (March 1933), Ultrashort-wave propagation, Proc. IRE 21. 429 463.

Smart, W. M. (1931), Spherical Astronomy, Chapter III (Cambridge University Press, London). 